\title{
Advanced Radio Resource Scheduling Algorithm for Energy Efficient Cellular Base Station
}

\author{
Hany M. Abdel-Aziz ${ }^{*}$, Fatma Newagy $^{\dagger}$, Nelly M. Shafik ${ }^{\ddagger}$, Ismail Hafez ${ }^{\S}$
}

\begin{abstract}
This work is to introduce an advanced calculation to enhance the energy efficiency of the radio base station for MU-MIMO-OFDM framework. Advanced Radio Resource Scheduling (ARRS) is introduced. Power utilization is considered a standout amongst the most imperative parts of the research for the wireless network. In view of the increasing mindfulness for saving the earth, and to give green frameworks. The calculations fundamentally divided into two phases. First, it discovers the resource shares, and the number of dynamic antennas in light of channel state based on essential Resource Allocations as Bandwidth Adaptation (BA) and Discontinuous Transmission (DTX). Secondly, it applies the Amplitude Carving Greedy (ACG) and Inverse Water Filling (IWF) Algorithms for the ARRS. Which finds the ideal number of resource blocks allocated to every client in view of the wanted rates, and the number of dynamic receiving antennas that maximize the utilization of base station power for the time changing frequency selective channel. The results illustrate that the proposed ARRS Algorithm is giving an upgrade in the diminishment of the supply power utilization from $10 \%$ in low rates up to $17 \%$ in high rates, compared with essential asset designation for RAPS calculations.
\end{abstract}

Keywords: Green wireless network, energy efficient, radio resource management, OFDMA, MIMO.

\section{Introduction}

The evolution of the wireless communication technologies has become enormous lately. Moreover, the usage of these systems has increased rapidly. Furthermore, the demand to access the internet to get any information has become huge. As result, the wireless communication section has become on high demand.

The carbon foot print has been a factor to measure the global warming based on the increased emission of $\mathrm{CO} 2$. Moreover, the power generation has been considered as one of the primal sectors to increase the greenhouse gas (GHG) in our atmosphere. Furthermore, the wireless communication section has become one of the rising sections in power consumption.

Teacher Assistance, Electronics \& Communication Eng. Department, Modern Academy for Engineering and Technology, Cairo, Egypt; eng.hany.mohamed84@gmail.com

$\dagger$ Assistant Professor, Electronics \& Communication Eng. Department, Ain Shams University, Cairo 11519, Egypt; fatma newagy@eng.asu.edu.eg

* Assistant Professor, Electronics \& Communication Eng. Department, Modern Academy for Engineering and Technology, Cairo, Egypt; nelly_mohammed@yahoo.com

$\S$ Professor, Electronics \& Communication Eng. Department, Ain Shams University, Cairo 11519, Egypt; ismail_hafez@eng.asu.edu.eg 
On the other hand, uprising awareness for our plant leads to the term "Green Technology", which is concerned about proposing the technology with minimum impact on the environment. One of the new trends is self-sufficient standalone Base Station (BS) that is used to minimise the mobile network power consumption [1].

There are different basic power saving techniques; Radio resource management (RRM) techniques like power control (PC), which is the most known technique, because it has advantages in linking adaptation and interference minimization, besides reducing consumption of the power as mentioned in [2], [3]. Other researchers investigate the field of the Antenna Adaptation and sleep mode for the long Term Evolution (LTE) as given in [4], [5]. Other researchers work on the inverse water filling is examined in [3], [6] - [8]. Discovering the suboptimum answer for the transmission control per client, including the transmission control utilization, the bit limit, and the adjustment for MIMO framework, which is examined in [3]. While another examination is done to discover suboptimum arrangement by keeping up reasonableness oblige, which is discussed in [6]. Other work in summing most of these techniques is the algorithm of Resource allocation using Antenna adaptation, Power control and Sleep mode (RAPS) in [8].

In this paper, we present new calculation, which studies the essential resource allocation strategies; the assignment of the resource blocks with the consideration of active sleep mode under the presumption of channel information.

This study focuses on the following: Segment II shows the power demonstrations and the framework display that are utilised. Segment III defines the problem under investigation. Segment IV demonstrates the steps for the proposed Algorithm which consist of two phases. The initial phase of the proposed algorithm ARRS, which is utilised for solving the problem to discover the initial values for the resource share and number of dynamic RF chains. and the second step of ARRS that helps in finding the best effort quantities of resource blocks for every client and number of dynamic RF chains and the aggregated power utilizations that is displayed. As for segment V, it explains the different results of the simulation case studies. Segment VI defines our conclusions.

\section{The Models of System and Power}

\section{A. Model of the System}

In this model, as explained in [8], we consider downlink transmission outline for indicated multipoint remote correspondence framework. In this model, there is a solitary base station that gives administration to a few cell phones. We consider applying the MIMO procedure, as the BS has $M_{T}$ Transmitter antennas and the recipients have $M_{R}$ Receiving antennas. The orthogonal frequency division Multiple Access (OFDMA) is utilized to share the resource shares. K, N, and T are Users, Subcarriers and Time Slots individually. For every resource block, $H_{n, t, k}$ with $M_{T} \times M_{R}$, assuming frequency selective time variant channel. The frame structure is shown in Fig.1 [8] and the framework is considered interference free. 


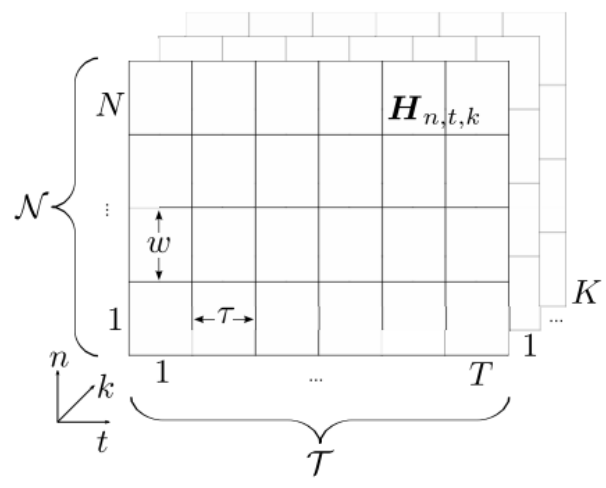

Fig. 1 OFDM frame structure [8]

\section{B. The Model of the Power}

The verified power models are used to calculate the total power consumption of the cellular BS in [9] and [10]. The supplying of power to the RF transmission is allowed by these models. The model of power in [11] is made from the implementation of the actual BS hardware, which has been verified by a detailed hardware model. A linear power model is used in this paper, which gives good basics for the analysis of the BS energy efficiency. Whereas, the power model of [9], [10] where the total supply power for the BS consists of the power consumed by base-band (BB) circuits, DC and AC conversation circuits, cooling system and RF chains. Each RF chain is considered as the combination of power amplifier (PA), small signal transceiver and transmitting antennas. The total supply power as given in [11]

$P_{\text {Total }}=\left(P_{P A}+P_{R F}+P_{B B}+P_{D C}+P_{A C}+P_{\text {cool }}\right)$.

Then the total BS Power consumption is giving by

$P_{\text {supply }}=M_{\text {sec }}\left(P_{\text {Total }}\right)$

where, $\mathrm{M}_{\mathrm{sec}}$ is the Sectors number for given cell, $\mathrm{P}_{\text {Total }}$ is the total consumption for the single sector. It is demonstrated that the greatest power consumption is in the Power Amplification (PA) area as appeared in [9]; and the framework is utilizing single antenna for every RF Chain. The antenna Adaptation (AA) is utilized to change the number of antennas in light of the requested rates. We could shut down the RF chains or place them in sleep mode independently based on the required QOS.

In this model, changing the RF chains status has no postponement to influence the transmission time, which is checked in [12].

In power model [9], the parameters are accommodated macro BS with one sector, and there is altered power that is utilized for the whole $\mathrm{BS}$, which is equivalent to least dynamic power utilization $\mathrm{P}_{o, M t}$, in view of the quantity of dynamic RF chains ; in this manner the aggregated power is fluctuating as indicated by the dynamic load utilized by the client, which relies on upon straight transmission power reliance considering $\Delta_{p m}$ and the power utilization amid the sleep mode $\mathrm{P}_{s}$. The power utilization for transmission at whatever time slot is $\mathrm{P}_{t}$.

The aggregated power utilization for one sector is given by

$P_{\text {supply }}\left(P_{t}\right)=\left\{\begin{array}{ll}P_{o, M t}+\Delta_{p m} \cdot P_{t} & \text { if } P_{t}>0 \\ P_{S} & \text { if } P_{t}=0\end{array}\right\}$. 


\section{Formulation of Problem}

In this section, a brief discussion of the different power saving techniques and the minimizing issue for the aggregated OFDMA supply power is proposed.

\section{Strategies of Power Saving}

There are three strategies that impact the utilization of BS power, the transmitted power $\mathrm{P}_{t}$, the dynamic number of RF chains $\mathrm{M}_{t}$ and the span time for which the BS enters the sleep mode as shown in Fig. 2 [9]. The three fundamental saving techniques are:

a. Power Control (PC): it means minimizing the transmission control for every resource block utilized.

b. Antenna Adaptation (AA): it intends to diminish the quantity of the antennas.

c. Discontinuous Transmission (DTX): it does not intend to transmit for all the time frame yet tries to increase the time spend in sleep mode.

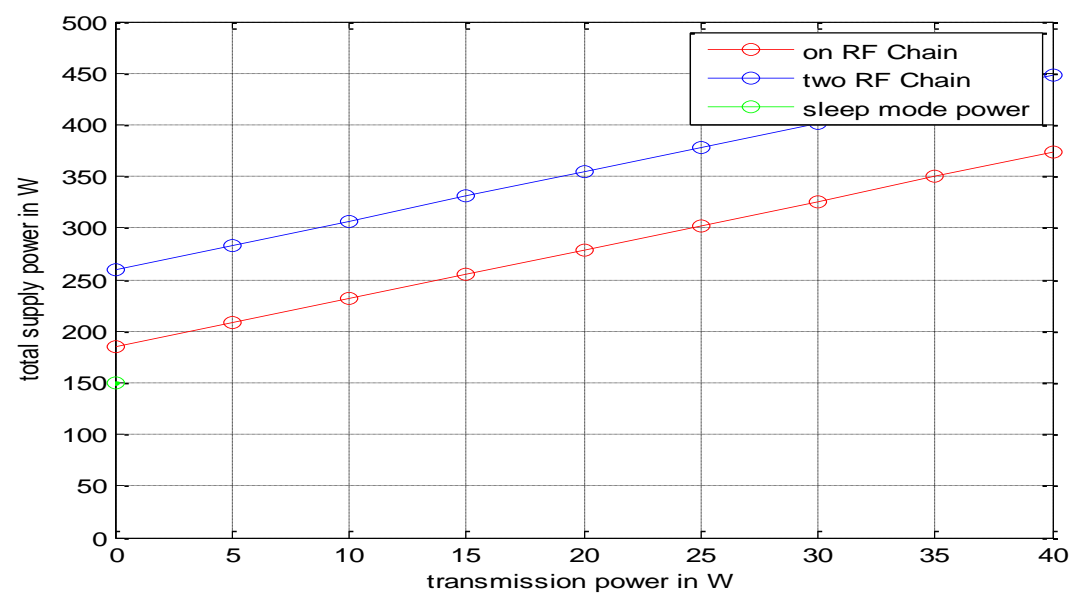

Fig. 2 Comparison between Different RF Chain Power Consumption. [9]

\section{Global Problem}

The problem of the power consumption is defined first by accepting that the channel state Matrices $H_{n, t, k}$ which is known for each channel and the objective rates per users $\left(R_{1}, R_{2}, \ldots\right.$ .., $R_{k}$ ). The arrangement of resource blocks that have been apportioned to every client is $A_{K}$. The power level for every resource Block is $\mathrm{P}_{\mathrm{a}, \mathrm{e}}$, the spatial channel is $\mathrm{e}=1, . . \varepsilon_{\mathrm{a}, \mathrm{k}}$ and active number of antennas number is $\mathrm{M}_{\mathrm{T}}$. The most extreme power transmission is $\mathrm{P}_{\max }$. Thus, the aggregated limit with respect to any client $\mathrm{k}$ on transmission frame duration $\mathrm{T}_{\text {frame }}$ is giving by

$C_{k}=\frac{w t}{T_{\text {frame }}} \sum_{a=1}^{A_{k}} \sum_{e=1}^{\varepsilon_{a, k}} \log _{2}\left(1+\frac{P_{a, e^{*} \varepsilon_{a, k}(e)}}{N_{o} w}\right)$.

where $w$ is the subcarrier bandwidth in $\mathrm{Hz}, \mathrm{t}$ is the duration of time slot in seconds, and $\mathrm{N}_{\mathrm{o}}$ is the density noise spectral in W/Hz. The RF transmission power for any time slot is

$$
P_{t}=\sum_{a=1}^{A_{T}} \sum_{e=1}^{\varepsilon_{a}} P_{a, e}
$$

where $\varepsilon_{\mathrm{a}}$ is the channel eigenvalues on resource $\mathrm{a}, \mathrm{A}_{\mathrm{T}}$ is the set of subcarrier assigned for any user k, From (3), (4) and (5) the problem is to minimize the total supply power as:

$P_{\text {supply }}(r)=\frac{1}{T}\left(\sum_{t=1}^{T_{\text {Acive }}}\left(P_{o, M t}+\Delta_{p m} P_{t}\right)+\sum_{t=1}^{T_{\text {sleep }}} P_{s}\right)$

Subject to $\mathrm{P}_{\mathrm{t}} \leq \mathrm{P}_{\max }, \mathrm{T}=\mathrm{T}_{\text {active }}+\mathrm{T}_{\text {sleep }}=$ frame duration. 
In addition, the rates for each user:

$$
R_{k}=\frac{w t}{T_{\text {frame }}} \sum_{a=1}^{A_{k}} \sum_{e=1}^{\varepsilon_{a, k}} \log _{2}\left(1+\frac{P_{a, e * \varepsilon_{a, k}(e)}}{N_{o} w}\right)
$$

where $\mathrm{T}_{\text {active }}$ is the active number of time slots, $\mathrm{T}_{\text {sleep }}$ is the number of time slot in sleep mode.

The assignment process of the Resource Blocks powerfully is known to be exceptionally mind boggling strategy for the subcarriers of every time slot and frequency selective channels, which is determined by means of computational costly calculations as specified in [2], [13], and [14]. Consequently, in this paper, the issue has been partitioned into two phases similar to [8]. In the first phase, the estimation of the Resource shares for every client $\mu_{\mathrm{k}}$, the quantity of sleep mode time slots, and the quantity of Active RF chains computed based on two techniques Bandwidth Adaptiation (BA) And Discontinuous Transmission (DTX). Secondly, the power utilization is resolved for the arrangement of doled out subcarriers and applying Amplitude Carving Greedy ACG And inverse water filling (IWF) power allocation until the ideal power utilization is accomplished.

\section{The Proposed ARRS Algorithm}

\section{A. Step 1: Finding the Estimates Parameters}

This section is attempting to focus of the finding the initial values for solving our problem using the proposed framework presumptions. First, it is confirmed to assume that each resources confront the same block fading instead of frequency selective fading as the middle Resource Block as in [8].

The equivalent power pre-coding and uncorrelated receiving antennas are utilized to recognize the connection capacity. The objective rate $R_{k}$ in the downlink block fading multiuser is given by

$R_{k}=W \mu_{k} \sum_{e=1}^{\varepsilon_{k}} \log _{2}\left(1+\frac{P_{k} \varepsilon_{k}(e)}{M_{T} N_{o} W}\right)$

where the $\varepsilon_{\mathrm{k}}$ is the channel eigenvalues per user $\mathrm{k}, \mathrm{P}_{\mathrm{k}}$ is the transmission power and $\mu_{\mathrm{k}}$ is resource share time $=\mathrm{t} / \mathrm{T}_{\text {frame }}$.

In addition, in the proposed system, the receiving antennas $M_{R X}$ equal two. From the transmission power computation and the power model in [8], the BS after serving the clients can go into sleep mode.

At this stage, we distribute the resource blocks among the users by two resource allocation techniques like bandwidth adaptation (BA) which transmits each resource block with the same power level $\mathrm{P}_{\max } / \mathrm{N}$ or by using the discontinuous transmission (DTX) which tries to transmit with the max power but in the same time minimize the number of time slots used to transmission and increase the sleep mode time.

\section{B. STEP2: Optimum Allocation of Power for ARRS}

The second phase of the ARRS is exhibited in this section of the study. The calculated estimations of step 1 are utilized to achieve the best effort values for the resource blocks for every client, while maintaining the same QoS. 
At first it tries to quantize the resource shares from step1 and the $\mathrm{T}_{\text {active }} \mathrm{TS}$.

At that point, the genuine esteemed asset share $\mu_{\mathrm{k}}$ is changed over into the OFDMA asset number per client $\mathrm{m}_{k} \leq \mathrm{N}$,

$$
\begin{aligned}
& m_{k}=\left\lceil m_{k} N T_{\text {active }}\right\rceil \quad \forall k=1, \ldots \ldots, K . \\
& m_{\text {rem }}=N T-\sum_{k=1}^{K} m_{k}-N T_{\text {sleep }} .
\end{aligned}
$$

The remaining unassigned resources $\mathrm{m}_{\mathrm{t}, \text { rem }}$ are conveyed between various clients in round robin design, while the availabilities considered for sleep mode is relegated to the end of the edge statically.

$m_{t, r e m}=N-\sum_{k=1}^{K} m_{k, t}$.

After that we apply one of the greedy algorithms like Amplitude Carving Greedy (ACG) as subcarrier allocation calculation from Kivanc et al. [13], which is demonstrated to give great results with low complexity. The initial step is to apply all the data we created like the subcarrier blocks, the quantity of dynamic RF chains and the quantity of sleep mode $\mathrm{T}_{\mathrm{S}}$ which are utilized to ascertain the transmit power in the area of time-frequency, and utilizing the inverse water filling (IWF), which works by first putting away the channels by their quality, and figuring the level of water of the best channel, that fulfils the bit load target. Furthermore, with every cycle, add the following best channel to the gathering of utilized channels, keeping in mind the end goal to decrease the water level. The calculation stops when the water level is less than the following channel metric to be utilized.

The target bit load for every client

$$
\beta_{\text {target }, k}=R_{k} T_{\text {frame }}
$$

To satisfy the target bit load, the next condition has to be maintained.

$\beta_{\text {target }, k}-\sum_{a=1}^{A_{k}} \sum_{e=1}^{\varepsilon_{a, k(e)}} w t \log _{2}\left(1+\frac{P_{a, e} \varepsilon_{a, k}(e)}{N_{o} w}\right)=0$

which ensures that the entirety limit with respect to the given gathering of channels and resource. The water level $v$ which will be computed in every cycle for gathering of channels $\Omega \_$k as takes after

$$
\log _{2}(\vartheta)=\frac{1}{\Omega_{k}}\left(\frac{\mathrm{B}_{\text {target }, k}}{w t}-\sum_{e=1}^{\Omega_{k}} \log _{2}\left(\frac{t \varepsilon_{a, k}(e)}{N_{o} \log (2)}\right)\right)
$$

Then, the power level per spatial channel

$P_{a, e}=\frac{\vartheta w t}{\log (2)}-\frac{N_{o} w}{\varepsilon_{a, k}(e)}$

The yield of this water filling calculation is the transmission power level $\mathrm{P}_{\mathrm{a}, \mathrm{e}}$ for every resource unit. Rehash the entire procedure until the quantity of free resource blocks is less than the quantity of resource blocks in a single TS (as $\mathrm{N}=50$ ). At the final stage, the figuring of the utilization of the supply power is finished by summing the transmission power for every resource block utilized. This is sketched out in the proposed ARRS is in Fig. 3 


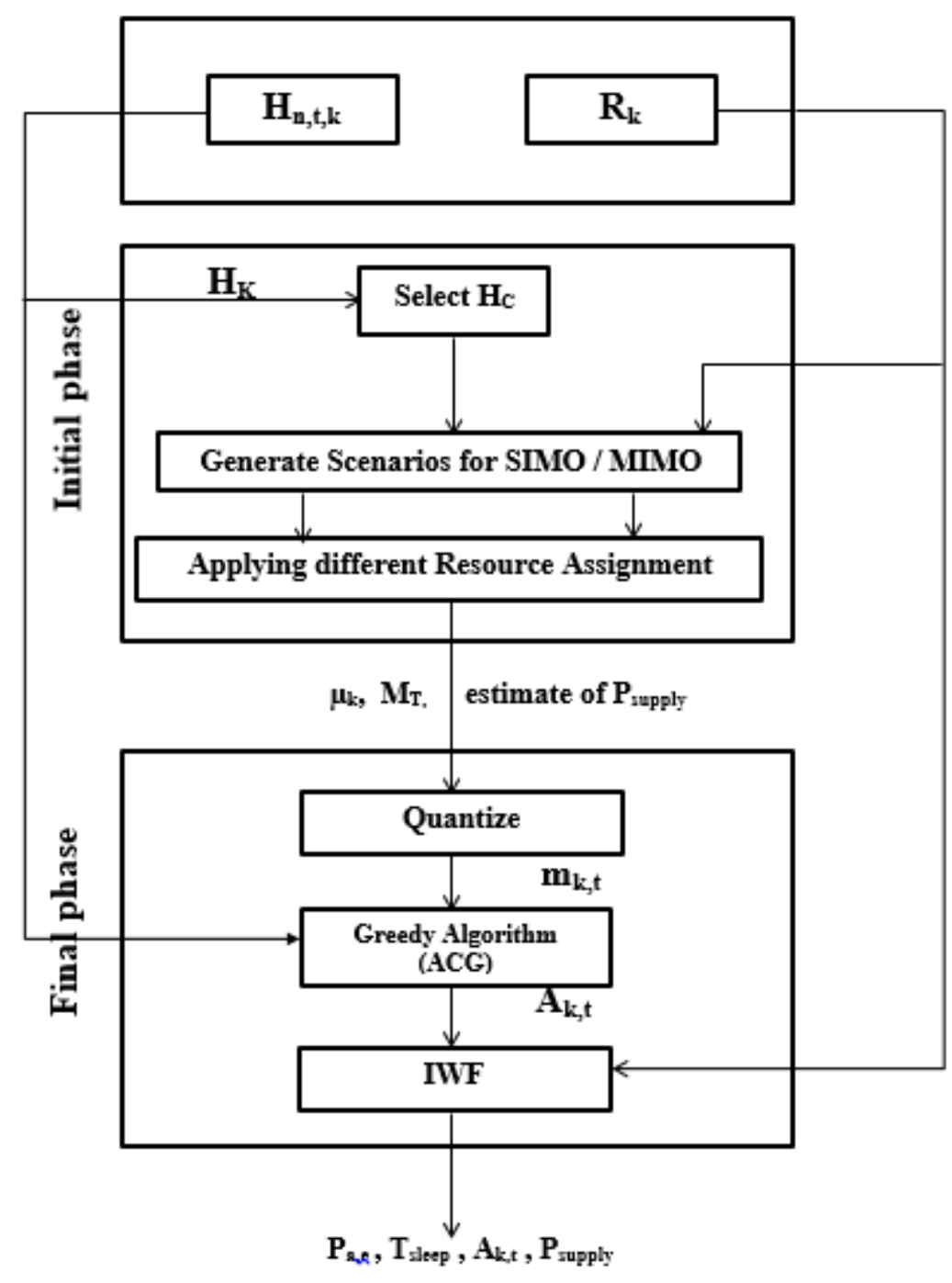

Fig. 3 The proposed Algorithm

Proposed Algorithm ARRS: Advanced Resource Allocation. The channel state indication $\mathrm{H}_{n, t, \mathrm{k}}$, the rate for each User $\mathrm{R}_{\mathrm{k}}$, the number of Active RF chains $\mathrm{M}$, the number of Users $\mathrm{K}$, and the number of time slots $T$. The resource shares $\mu_{\mathrm{K}}$ per user, the number of the sleep mode time slots $\mathrm{T}_{\mathrm{s}}$.

1: collect the rates vector $\mathrm{R}_{\mathrm{K}}=\mathrm{R}_{1}, \mathrm{R}_{2} \ldots \mathrm{R}_{\mathrm{k}}$.

2: collect the channel state information

$\mathrm{CSI}=\mathrm{H}_{\mathrm{n}}{ }^{\mathrm{c}, \mathrm{c}}{ }^{\mathrm{c}} \mathrm{k}$, for the Rf Chains.

3: preform resource allocation technique as (BA / DTX) and get estimates of $\left\{\mathrm{M}, \mu_{\mathrm{K}}\right.$, Psupply, $\mathrm{T}_{\mathrm{s}}$.

4: quantize the RBs over the $\mathrm{T}_{\text {active }}$.

5: apply greedy Algorithm (ACG) to assign each user with required RBs.

6: preform IWF to calculate the $\mathrm{P}_{\mathrm{tx}}$ for each $\mathrm{RB}$.

7: calculate the supply power for the system. 


\section{Simulation Results}

In order to test the ARRS Algorithm, Monte Carlo simulation is applied, as in [8] demonstrated with the accompanying parameters:

On hover with span $250 \mathrm{~m}$, the mobiles are consistently circulated around the BS, with least of $40 \mathrm{~m}$ to the BS. As indicated by the NLOS display depicted in 3GPP.TR25.814, selective fading channels is processed as in [15], where shadowing standard deviation is $8 \mathrm{~dB}$. The model of the recurrence specific divert BS depicted in [16] with $3 \mathrm{~m} / \mathrm{s}$ versatile speed. Each transmitter and receiving antennas is thought to be commonly uncorrelated.

The system parameters are in table.1.

Table 1 System parameters

\begin{tabular}{|c|l|c|}
\hline Variable & \multicolumn{1}{|c|}{ Name } & Value \\
\hline $\mathrm{K}$ & Number of users & $10-20$ \\
\hline $\mathrm{N}$ & Number of subcarriers & 50 \\
\hline $\mathrm{T}$ & Number of time slots & 10 \\
\hline $\mathrm{M}_{\mathrm{T}}$ & Number of transmit antenna & {$[1,2]$} \\
\hline $\mathrm{M}_{\mathrm{R}}$ & Number of receiver antenna & 2 \\
\hline $\mathrm{P}_{\mathrm{o}, \mathrm{MT}}$ & Circuit power consumption & $185 \mathrm{~W}, 260 \mathrm{~W}$ \\
\hline$\Delta_{\mathrm{pm}}$ & Load dependence factor & 4.7 \\
\hline $\mathrm{P}_{\mathrm{s}}$ & Power consumption in DTX & $150 \mathrm{~W}$ \\
\hline $\mathrm{P}_{\mathrm{max}}$ & Maximum transmission power & $46 \mathrm{dBm}$ \\
\hline $\mathrm{T}_{\mathrm{frame}} / \mathrm{t}$ & Duration of frame / time sot & $10 \mathrm{~ms} / 1 \mathrm{~ms}$ \\
\hline $\mathrm{W} / \mathrm{w}$ & System /subcarrier bandwidth & $10 \mathrm{MHz} / 200 \mathrm{KHz}$ \\
\hline $\mathrm{N}_{\mathrm{o}}$ & Noise power spectral density & $4 \times 10^{-21} \mathrm{~W} / \mathrm{Hz}$ \\
\hline
\end{tabular}

Keeping in mind the end goal to look at the outcomes, the accompanying transmission techniques are performed:

- $\quad$ Maximum BS power utilization, where the BS has settled transmission power $\mathrm{P}_{\max }$.

- $\quad$ Bandwidth Adaptation (BA), where it tries to locate the base number of subcarriers that accomplish the objective rate and the sleep mode is not activated and all channels transmit with power equivalent to $\mathrm{P}_{\max } / \mathrm{N}$.

- Discontinuous transmission (DTX), where BS transmits with $\mathrm{P}_{\max }$ and go into sleep mode when the objective rate accomplished.

Figure 4 shows that the DTX method is more effective than the BA; as it tries to free some Time slots. The BS in sleep mode, however, it is as yet consuming much power. Henceforth, the work is done on the others strategies like RAPS and the proposed method ARRS.

The examination begins for the recommended algorithm ARRS versus the RAPS and the hypothetical maximum. Fig.5 reveals that those new calculations in the high rate area is giving $17 \%$ gain against the RAPS and $51 \%$ gain against the theoretical Maximum while the RAPS is giving $41 \%$ gain. Nonetheless, morals on low rate area gain is $10 \%$ compared to RAPS, while it gives $62 \%$ compared to the theoretical maximum and the RAPS is giving $58 \%$

While in Fig.6, the energy efficiency (bit/joule) is investigated; where,

$$
E=P_{\text {supply }}^{-1} \sum_{k=1}^{K} R_{k}
$$

For BA and DTX based ARRS assigned for the users on the overall energy efficiency. 


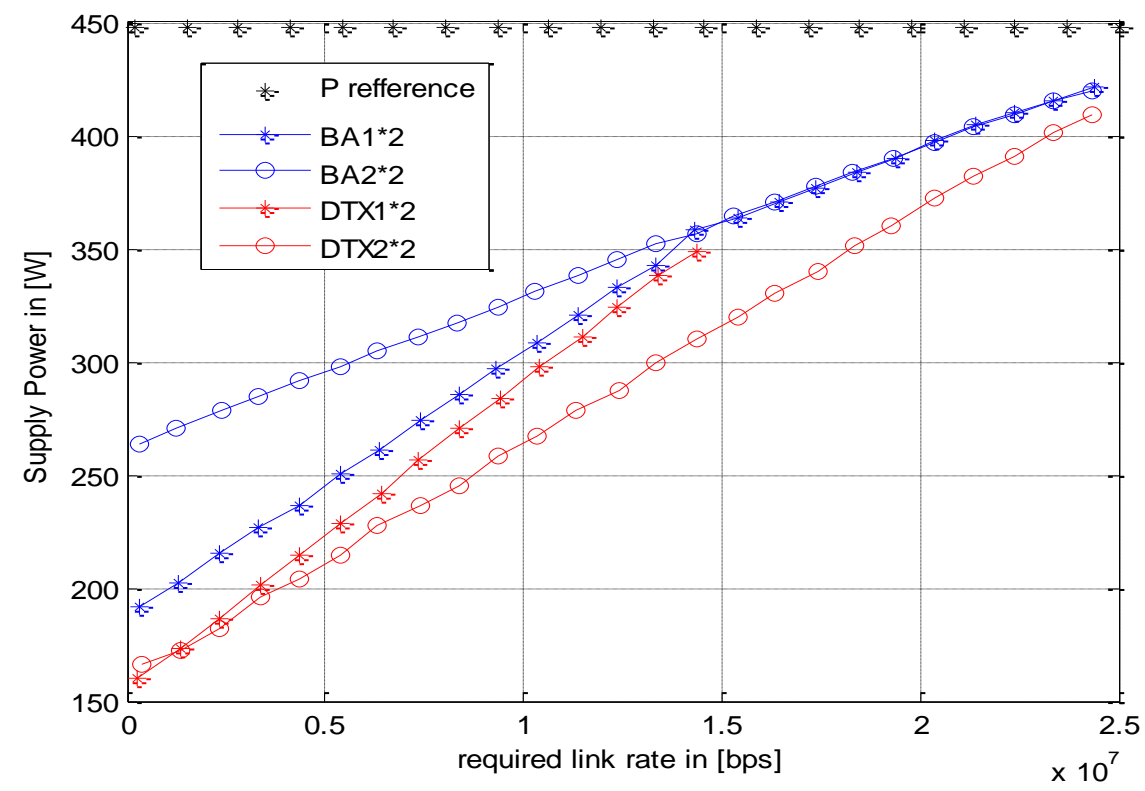

Fig. 4 Reference Power Consumption for Bench Mark Algorithm

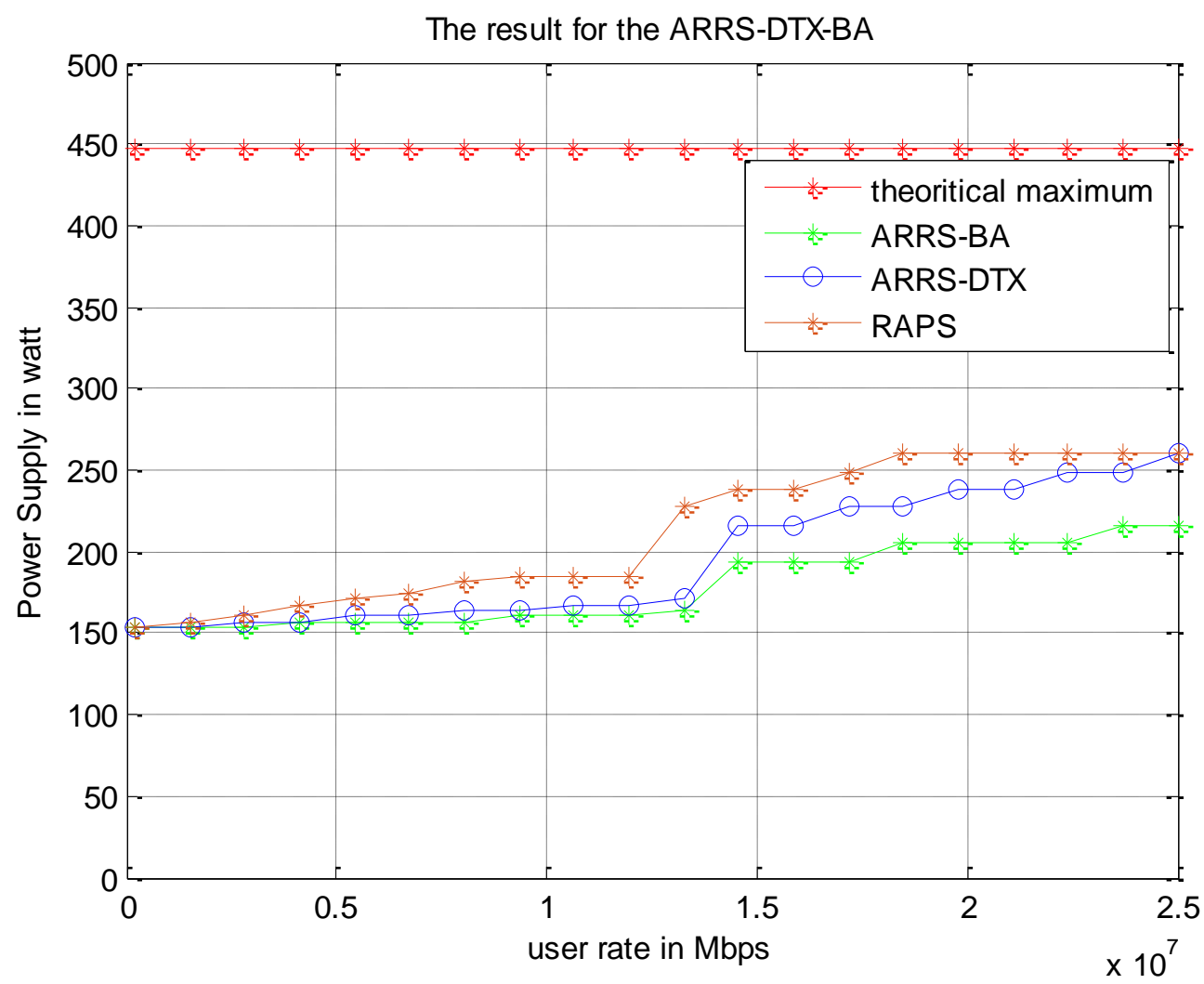

Fig. 5 Supply Power Consumption vs. the Requested Rates for Users 
The Efficiency in ARRS is superior to RAPS by $20.4 \%$ at most maximum summation rates and it is superior to the theoretical maximum by $107.35 \%$, while the RAPS is better by $72.23 \%$ compared to the theoretical maximum. And is also superior to the ARRS.

The proposed ARRS algorithm is providing better results with almost the same ration for different power models like the improved DTX and STOA 2010 as shown in Fig. 7 and Fig. 8.

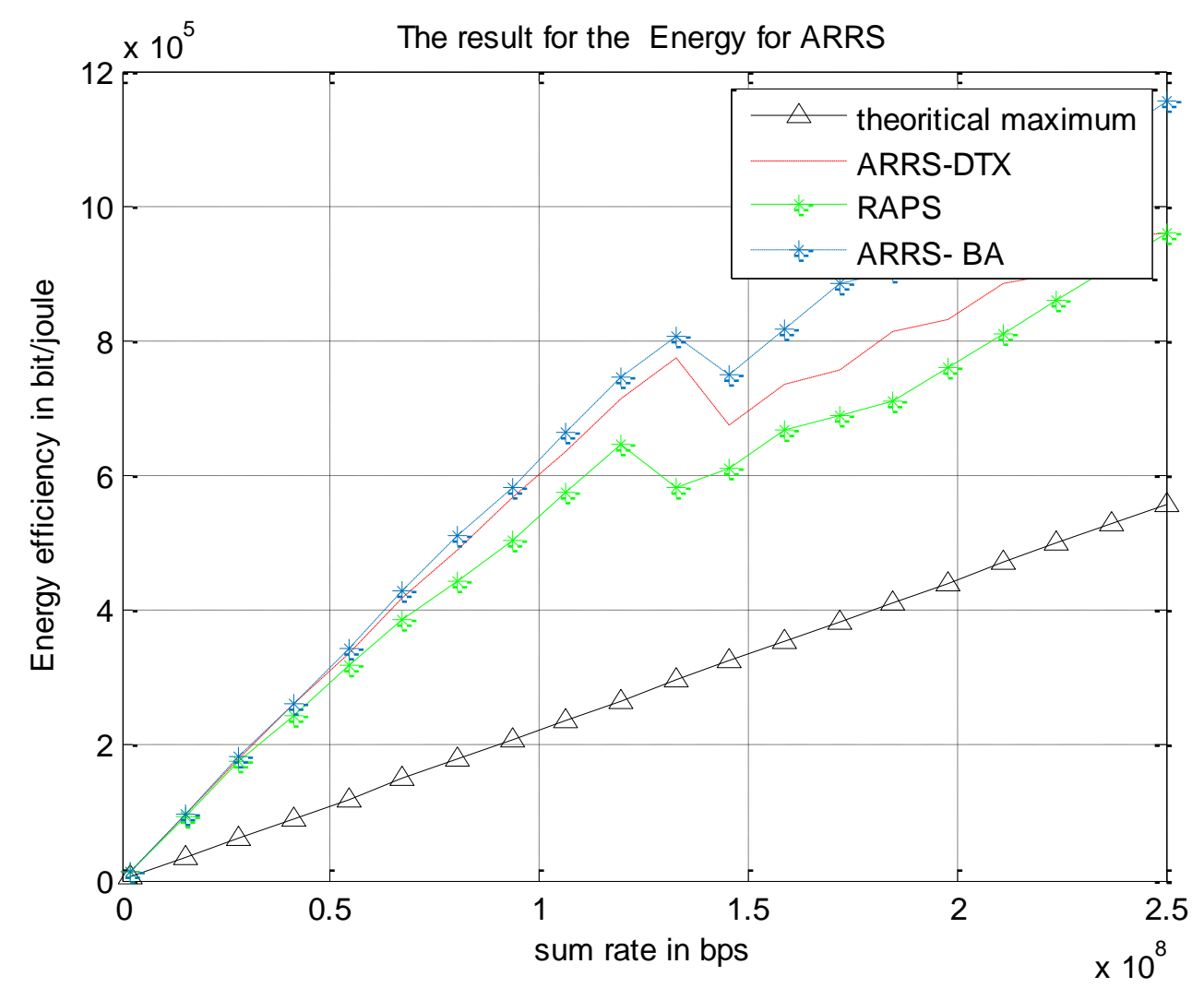

Fig. 6 Comparison between Energy Efficiency for both Resource Allocations

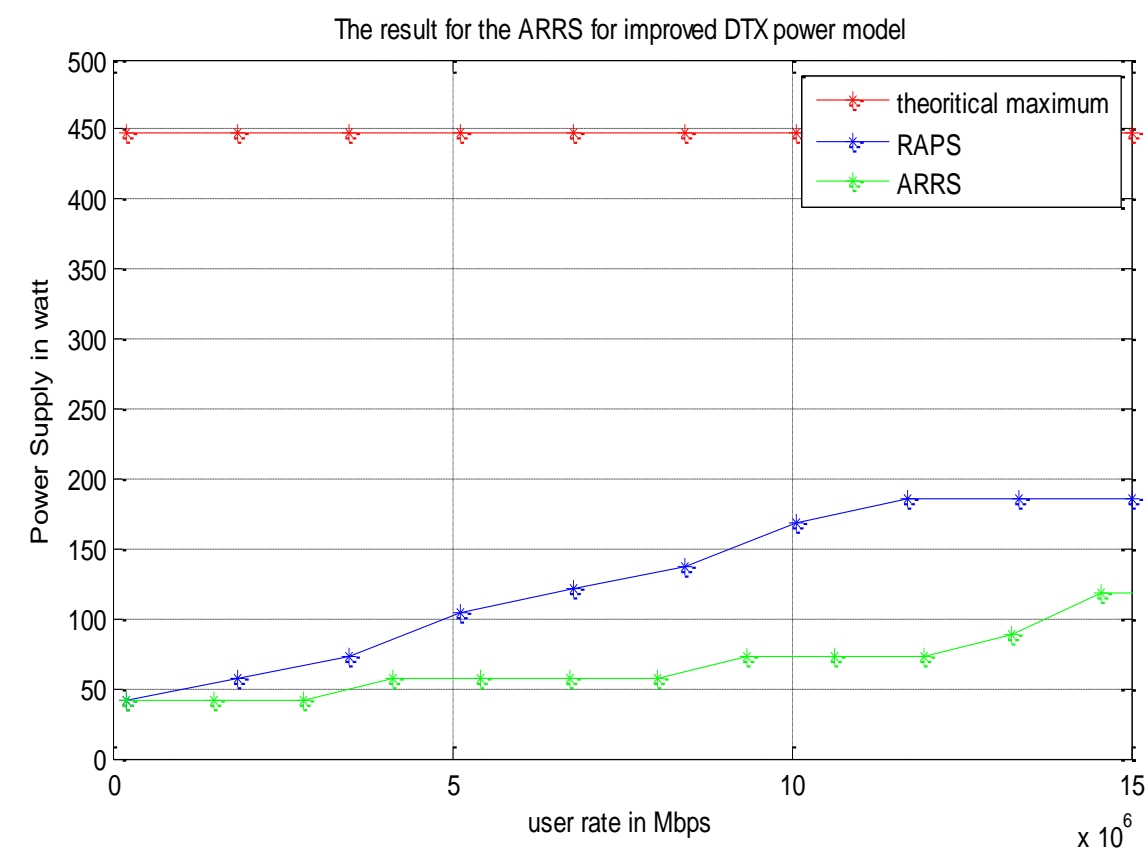

Fig. 7 The Result for the improved DTX Model. 


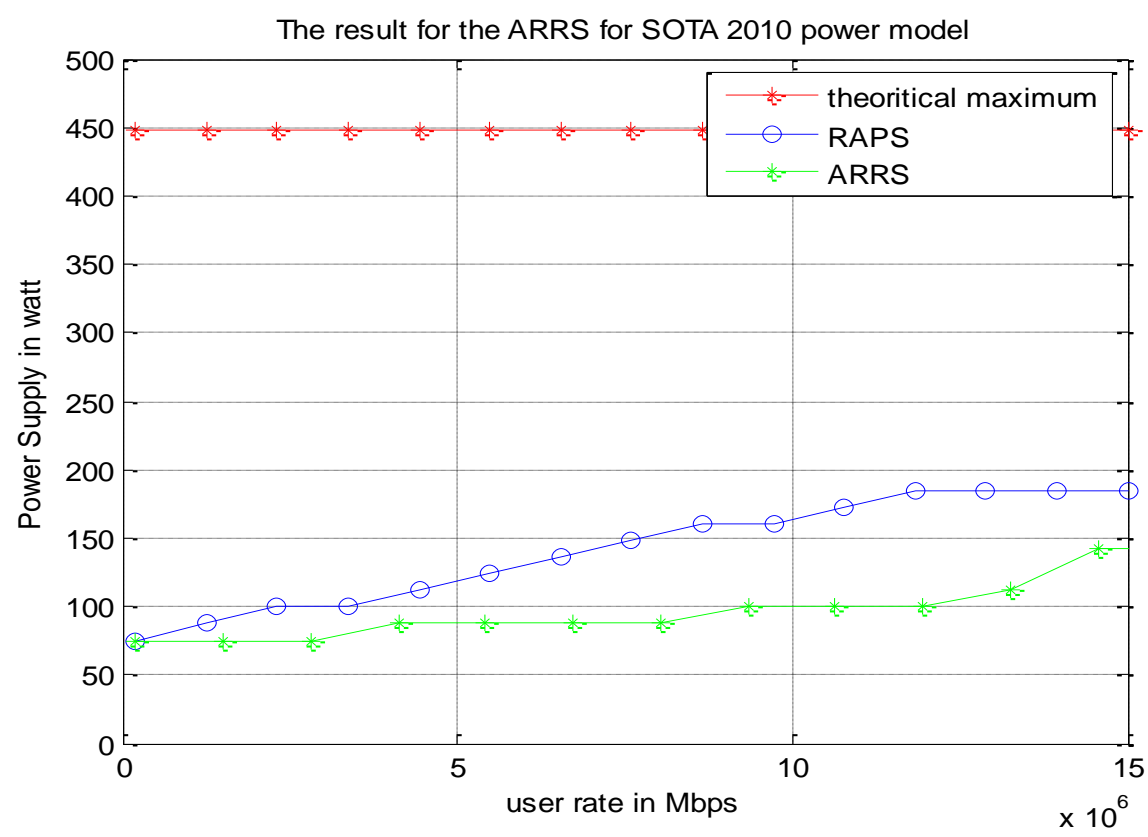

Fig. 8 The Result for the STOA 2010 Power Model

\section{Conclusion}

In this paper, BS resource Assignment methods like BA, DTX, and RAPS are studied and contrasted in regards to their execution and the proposed algorithm ARRS, we have thought about the framework display for multiuser MIMO OFDM. Reproduction results from demonstrating that the ARRS calculation is better in results than RAPS .also the ARRS is giving upgrade in lessening of supply power utilization from $10 \%$ in low rates to $17 \%$ in high rates; which is contrasted with RAPS calculation in [8].

And to $62 \%$ in low rates to $51 \%$ in high rates compared to the maximum power usages.

It also shows that while the DTX is giving better results than BA for power consumption, But our proposed algorithm is giving better result when depending on the BA as the first state in our algorithm than using the DTX.

\section{References}

[1] A. Fehske, J. Malmodin, G. Bicz'ok, and G. Fettweis, "The Global Carbon Footprint of Mobile Comunications - The Ecological and Economic Perspective," IEEE Commun. Mag., August 2011, pp 55-62.

[2] C. Y. Wong, R. S. Cheng, K. B. Lataief, and R. D. Murch, "Multiuser OFDM with Adaptive Subcarrier, Bit, and Power Allocation," IEEE J. Sel. Areas Commun., vol. 17, no. 10, Oct. 1999, pp. 1747-1758.

[3] S. Cui, A. J. Goldsmith, and A. Bahai, "Energy-Efficiency of MIMO and Cooperative MIMO Techniques in Sensor Networks," IEEE J. Sel. Areas Commun., Volume: 22, Issue: 6, Aug. 2004, pp1089-1098.

[4] M. Hedayati, M. Amirijoo, P. Frenger, and J. Moe, "Reducing Energy Consumption Through Adaptation of Number of Active Radio Units," in Proc. IEEE VTC, 2012-May 2011, pp. 1-6.

[5] R. Wang, J. Thompson, H. Haas, and P. Grant, "Sleep Mode Design for Green Base Stations," IET Communications, vol. 5, no. 18, 2011, pp. 2606-2616. 
[6] Z. Shen, J. Andrews, and B. Evans, "Optimal power allocation in multiuser OFDM systems," in Global Telecommunications Conference,2003. GLOBECOM'03. IEEE, vol. 1. IEEE, 2003, pp. 337-341.

[7] H. Al-Shatri and T. Weber, "Fair Power Allocation for Sum-Rate Maximization in Multiuser OFDMA," in Proc. International ITG Workshop on Smart Antennas 23-24 Feb. 2010, pp. 350-354.

[8] Holtkamp, H., Auer, G., Bazzi, S., \& Haas, H. Minimizing Base Station Power Consumption. IEEE Journal on Selected Areas in Communications, vol. 32, no. 2, 2014, pp 297-306.

[9] G. Auer, V. Giannini, I. G’odor, P. Skillermark, M. Olsson, M. Imran, D. Sabella, M. J. Gonzalez, and C. Desset, "Cellular Energy Efficiency Evaluation Framework," in Proc. VTC 2011-15-18 May, 2011, pp. 2418-2423.

[10] G. Auer, V. Giannini, I. G'odor, P. Skillermark, M. Olsson, M. A. Imran, M. J. Gonzalez, C. Desset, O. Blume, and A. Fehske, "How Much Energy is Needed to Run a Wireless Network?" IEEE Wireless Commun., vol. 18, no. 5, Oct 2011, pp. 40 -49.

[11] C. Desset, B. Debaillie, V. Giannini, A. Fehske, G. Auer, H. Holtkamp, W. Wajda, D. Sabella, F. Richter, M. Gonzalez, H. Klessig, I. G'odor, P. Skillermark, M. Olsson, M. A. Imran, A. Ambrosy, and O. Blume, "Flexible power modeling of LTE base stations," in 2012 IEEE Wireless Communications and Networking Conference: Mobile and Wireless Networks (IEEE WCNC 2012 Track 3 Mobile \& Wireless), 1-4 April 2012, pp. 2858-2862.

[12] P. Frenger, P. Moberg, J. Malmodin, Y. Jading, and I. G'odor, "Reducing Energy Consumption in LTE with Cell DTX," in Proc. IEEE VTC 2011, 15-18 May 2011, pp. $1-5$.

[13] D. Kivanc, G. Li, and H. Liu, "Computationally Efficient Bandwidth Allocation and Power Control for OFDMA," IEEE Trans. Wireless Commun., vol. 2, no. 6, Nov. 2003, pp. 1150-1158.

[14] J. Jang and K. B. Lee, "Transmit Power Adaptation for Multiuser OFDM Systems," IEEE J. Sel. Areas Commun., vol. 21, no. 2, Feb.2003, pp. 171-178.

[15] 3GPP, "Physical Layer Aspects for Evolved Universal Terrestrial Radio Access (UTRA)," 3GPP TR 25.814 V7.1.0 (2006-09), Sep. 2006. Retrieved Nov. 2, 2009 from www.3gpp.org/ftp/Specs/.

[16] IST-2003-507581 WINNER, "D5.4 v1.0 Final Report on Link Level and System Level Channel Models," Retrieved Apr. 15, 2007, from https://www.ist-winner.org/DeliverableDocuments/, Nov. 2005. 